Adolesc Psychiatry Nov 2008;47:1321-1328). (Repond: Amy Garrett PhD, Department of Psychiatry, 401 Quarry Rd, Stanford, CA 94305. E-mail: agaarrett(a) stanford.edu).

COMMENT. Neuroanatomical abnormalities detected in patients with ADHD support the neurobiological basis for this symptom complex. The authors cite a meta-analysis of structural imaging research that indicates regions most frequently affected include total cerebral volume, caudate nucleus, splenium of the corpus callosum, cerebellum and frontal lobes. Differences in findings may be attributable to differences in age of subjects selected and the weight of hereditary compared to environmental causes of ADHD (Millichap JG. Etiological classification of attention-deficit/hyperactivity disorder. Pediatrics 2008;121:e358-e365). The selection of cases with familial ADHD by the Stanford group is a more homogeneous sample than some, emphasizing the neural correlates of inherited forms of the disorder. The possible influence of associated environmental factors is not excluded, however.

\title{
ADHD SYMPTOMS AND LIKELIHOOD OF CHILD MALTREATMENT
}

The relationship between inattentive and hyperactivity symptoms and child maltreatment was studied among a sample of 14,322 participants in the National Longitudinal Study of Adolescent Healh at the Centers for Disease Control and Prevention, Atlanta, GA. The weighted percentage of respondents reporting ADHD symptoms was $8.3 \%$. Self-reported rates of child maltreatment were $40.5 \%$ for supervision neglect, physical neglect $(11.6 \%)$, physical abuse $(28.4 \%)$, and contact sexual abuse $(4.5 \%)$. Forty six percent reported no child maltreatment. The age of subjects sampled was 18 to 28 years (average 21.8 years). Type of maltreatment was not correlated with age. Respondents with ADHD symptoms were more likely to report maltreatment. Compared with non-ADHD subjects, those with ADHD of any type reported all 4 types of child maltreatment. The inattentive type was associated with elevated risks of all 4 types of maltreatment whereas the hyperactive/impulsive type was associated only with an increased likelihood of supervision neglect and physical abuse. The combined type was associated with elevated risks of physical neglect and contact sexual abuse, and a significant risk of supervision neglect. The number of reported ADHD symptoms was also associated with the severity of child maltreatment. Each additional inattentive symptom was significantly associated with elevated risks of more severe child maltreatment of all 4 types. Each additional hyperactive/impulsivity symptom was associated with an increased likelihood of more severe supervision neglcct or physical abuse. (Ouyang L, Fang X, Mercy J, Perou R, Grosse SD. Attention-deficit/hyperactivity disorder symptoms and child maltreatment: a population-based study. J Pediatr Dec 2008;153:851-856). (Reprints: Lijing Ouyang PhD, Centers for Disease Control and Prevention, 1600 Clifton Rd NE, Mail-Stop E-88, Atlanta, GA 30333. E-mail: eop9(a.cdc.gov).

COMMENT. Physicians should be alerted to the potential for child maltreatment in children with ADHD. Those with the inttentive type of ADHD are particularly vulnerable. The more severe the ADHD, the greater is the likelihood of child maltreatment. 